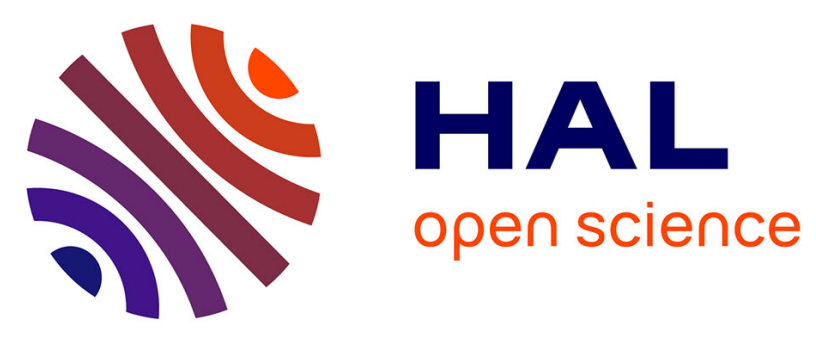

\title{
Optimal Work Space of Parallel Continuum Manipulator Consisting of Compact Bionic Handling Arms
}

\author{
Inderjeet Singh, Manarshhjot Singh, Pushparaj Mani Pathak, Rochdi
}

Merzouki

\section{- To cite this version: \\ Inderjeet Singh, Manarshhjot Singh, Pushparaj Mani Pathak, Rochdi Merzouki. Optimal Work Space of Parallel Continuum Manipulator Consisting of Compact Bionic Handling Arms. IEEE Conference on Robotics and Biomimetics (ROBIO 2017), Dec 2017, Macau, China. hal-01667911}

\section{HAL Id: hal-01667911 \\ https://hal.science/hal-01667911}

Submitted on 19 Dec 2017

HAL is a multi-disciplinary open access archive for the deposit and dissemination of scientific research documents, whether they are published or not. The documents may come from teaching and research institutions in France or abroad, or from public or private research centers.
L'archive ouverte pluridisciplinaire HAL, est destinée au dépôt et à la diffusion de documents scientifiques de niveau recherche, publiés ou non, émanant des établissements d'enseignement et de recherche français ou étrangers, des laboratoires publics ou privés. 


\title{
Optimal Work Space of Parallel Continuum Manipulator Consisting of Compact Bionic Handling Arms
}

\author{
Inderjeet Singh ${ }^{1}$, Manarshhjot Singh ${ }^{1}$, Pushparaj Mani Pathak ${ }^{2}$ and Rochdi Merzouki ${ }^{1}$
}

\begin{abstract}
Parallel continuum manipulators are the main research focus these days. Parallel manipulators have other advantages than serial manipulators in terms of acceleration and positioning. In this work, we proposed the use of two Compact Bionic Handling Arm (CBHA) manipulators, attaching them to a common platform, to constitute a parallel continuum manipulator. A relation is developed for optimal platform length as a function of the inter-distance between bases of two arms, by using optimization of the volume of the common work space realized from multiple CBHA. The work is extended to parallel continuum manipulator consisting of three CBHA manipulators. Same for this case, after identifying work space of this parallel continuum manipulator, a relation is developed between different design parameters of manipulator using optimization of volume of its work space. As the work space is the main limitation in case of parallel manipulators, this approach to generate relation is useful for rigid as well as continuum manipulators to design them for optimal work space as per required application.
\end{abstract}

\section{INTRODUCTION}

Parallel manipulators are best suited for high accuracy, high speed and acceleration applications. Since Stewart's contribution in the field of parallel robots, they are proved to be a strong supplement to serial robots. There are a lot of contributions in case of rigid parallel manipulators but there is very less work in case of parallel continuum manipulators. In [1] the kinematics of a hexa parallel machine is solved using geometrical formulation. Then work space analysis is done using kinematic model. Work space of a 3RPS parallel manipulator is computed using cylindrical algebraic decomposition method in [2]. [3] describes a parallel robot $3-\mathrm{R} 2 \mathrm{H} 2 \mathrm{~S}$ to meet the needs of sorting and packing in drug and food industries. After computing the kinematic model, its work space is being analyzed using boundary search method through MATLAB and ADAMS. In [4], the kinematics of a 3-PPPS parallel manipulator and its work space analysis is discussed using cylindrical algebraic decomposition method. 3-PPPS parallel manipulator is claimed as the simplest 6 DOF manipulator. A new parallel mechanism based on simulation driven design, which can generate three degree of freedoms is proposed in [5]. The work space is calculated for this mechanism using boundary searching method and then it is optimized using swarm algorithm. A novel 3-PUU parallel

\footnotetext{
${ }^{1}$ Authors are with Polytech Lille, CRIStAL, CNRS-UMR 9189, Avenue Paul Langevin, 59655 Villeneuve d'Ascq, France inderjeet.singh@ed.univ-lillel.fr manarshhjot.singhegmail.com

2 Department of Mechanical and Industrial Engineering, Indian Institute of Technology Roorkee, Roorkee 247667, India pushpfmeditr.ac.in
}

robot, its kinematics and work space using three dimensional limit search method are discussed in [6] and the work space is optimized using genetic algorithm.

All of the discussed work is on rigid parallel manipulators. In [7], work is started towards the direction of parallel continuum manipulators. In this work construction of a prototype manipulator with six compliant legs connected parallel to each other same as Stewart's platform is described. Kinematic solution of this manipulator is done using cosserat-rod models with coupled boundary conditions. A cone shaped parallel continuum robot is designed in [8], because of non linearity in the system, artificial neural network is used to compute kinematics of manipulator.

In this paper, a parallel continuum manipulator comprises from two as well as three Compact Bionic Handling Arms (CBHA) is proposed. Then its work space is calculated using kinematics. In addition to the above, another proposition is the relation between design parameters of the parallel continuum manipulator by optimizing the volume of the work space.

Reasons of moving from serial manipulator to parallel continuum manipulator:

- Load carrying capacity for parallel manipulator is higher than that of serial manipulator.

- CBHA being a continuum manipulator deforms during load handling. This deformation is reduced by using parallel configuration as the load is distributed among many arms. Therefore, accuracy in case of parallel continuum manipulator would be more.

- In case of parallel continuum manipulator, more orientations can be achieved than parallel rigid manipulator.

The paper is organized as follows: Section II describes one single CBHA manipulator and its working. Work space identification has been done in section III for single manipulator. Section IV is focused on the parallel continuum manipulator designed using two CBHA arms, its work space identification, optimization as well as results are discussed. Likewise, section $\mathrm{V}$ is extended to parallel continuum manipulator designed from three CBHA arms. Finally the work is concluded in section VI and also future aspects are discussed.

\section{COMPaCt Bionic Handling ARM}

Compact Bionic Handling Arm (CBHA) is a continuum manipulator made up of polyamide material. Material imparts property of flexibility and light weight to the manipulator. Fig. 1 represents the CBHA manipulator, it consists of a compliant gripper, a rotating part and two flexible sections. Each section consists of three tubes and each tube 
is actuated pneumatically. Pressure variation inside tubes results in the different postures of the manipulator. Equal pressures in all tubes of a section extends the whole section but the differential pressures are used to achieve bending. The total length of CBHA manipulator is restricted using a non extensible cable at the backbone of CBHA. To measure the lengths of the tubes, potentiometer wires are attached on the tubes. Lengths $l_{i, j}$ can be calculated proportional to the voltage recorded from potentiometers. Here $i=1, \ldots, 3$ refers to the number of tube and $j=1,2$ corresponds to the section number.

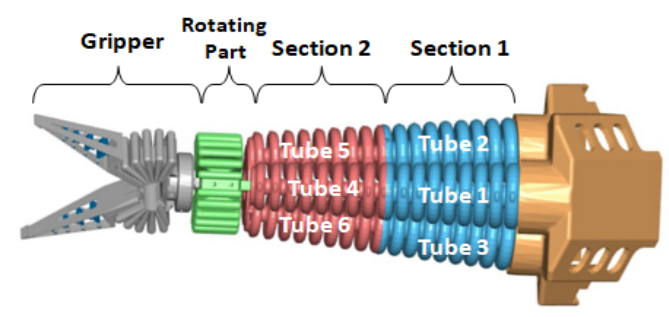

Fig. 1. CBHA Manipulator

\section{Work Space IDENTIFICATION FOR Single CBHA MANIPULATOR}

Work space is identified for one single manipulator, then a setup from two as well as three CBHA manipulators is considered to make parallel continuum manipulator and the work space is identified for parallel manipulator. Work space identification for single manipulator is as follows:

Work space of CBHA manipulator covers the space in which tip of the manipulator can reach. The work space is calculated using Forward Kinematic Model (FKM) of CBHA manipulator, which is developed in [9] using arc geometry method. Fig. 2 shows the CBHA manipulator with frame

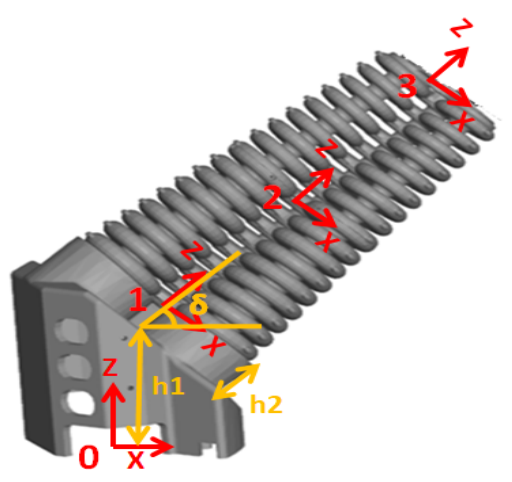

Fig. 2. CBHA manipulator with frames

assignment. Fig. 3 represents the simulated work space with the variation of all possible tube lengths. To verify simulated work space, an experimental work space is also computed using experimental set-up as in Fig. 4. All possible set of pressures are applied to CBHA manipulator. Stereo vision
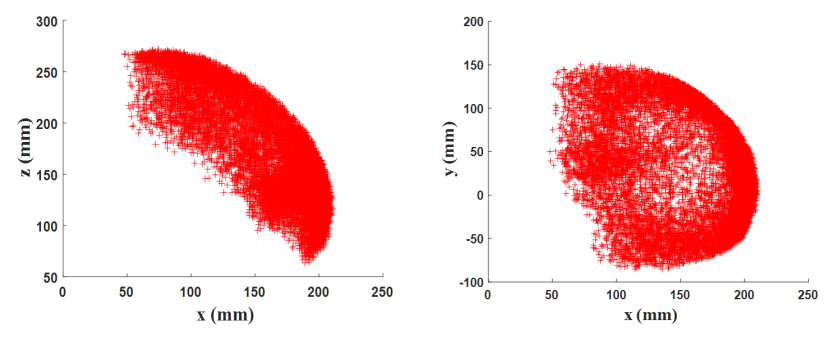

Fig. 3. Simulated work space of single CBHA manipulator

system is used to track tip point of manipulator. For stereo vision, configuration of camera is: Basler acA645-100gc with the sony ICX414 CCD sensor. The camera delivers 100 frames per second at $(658 \mathrm{px} \times 492 \mathrm{px})$ resolution. Aruco markers are used for detection of tip point of CBHA manipulator as shown in Fig 4. Experimental work space is plotted as in Fig. 5, it is verified that the shape of this work space is same as simulated work space.
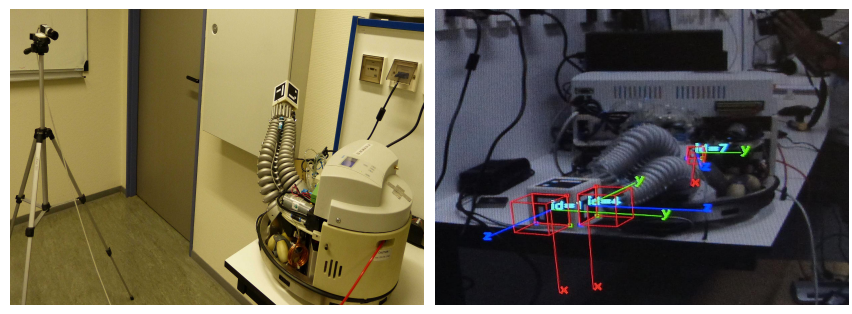

Fig. 4. Experimental set-up for work space calculation

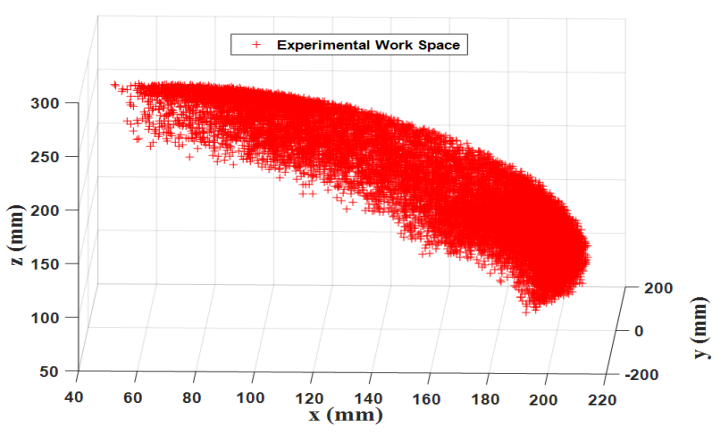

Fig. 5. Experimental work space for single CBHA

\section{Parallel Continuum Manipulator Designed USING TwO CBHA ARMS}

\section{A. Work Space Identification}

A parallel continuum manipulator can be created using two CBHA arms placing at a distance of $D_{p}$ and a common platform of length $a$ attached on the tips of both of the arms through spherical joints as shown in Fig. 6. For any application, it is important to find out the work space of the center point of the platform, it can be either used to handle objects or an gripper can be attached on the center point for 
pick and place purposes. The work space of the center point of the platform should be a common work space for both of the arms, where both of them can reach. Therefore firstly, work space for both of the arms is generated till their tip points. Then these are extended to the center point $p$ of the platform using transformations. Transformation till center of platform for first arm is done as:

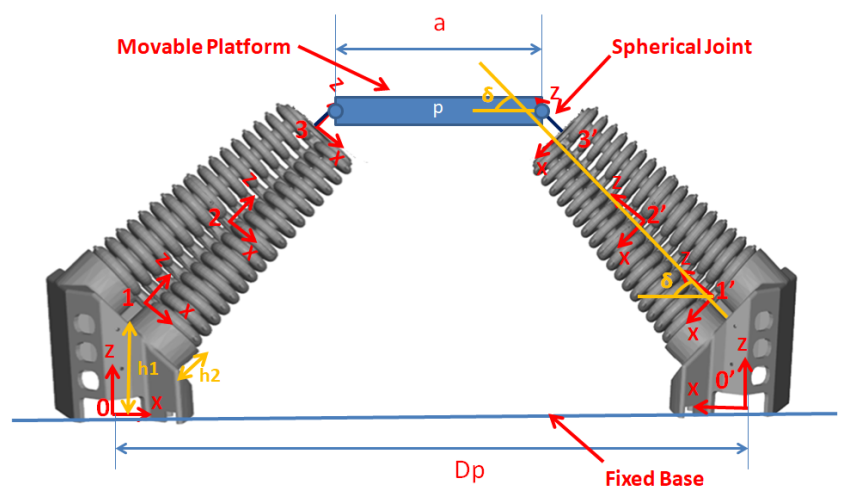

Fig. 6. Set-up for parallel continuum manipulator using two CBHAs

$$
{ }_{p}^{0} T={ }_{1}^{0} T_{2}^{1} T_{3}^{2} T_{p}^{3} T
$$

Here ${ }_{p}^{3} T$ is,

$$
{ }_{p}^{3} T=\left[\begin{array}{cccc}
\cos \delta & 0 & \sin \delta & 0 \\
0 & 1 & 0 & 0 \\
-\sin \delta & 0 & \cos \delta & a / 2 \\
0 & 0 & 0 & 1
\end{array}\right]
$$

For second arm, transformations are taken from the same origin frame 0 .

$$
{ }_{p}^{0} T={ }_{0^{\prime}}^{0} T_{1^{\prime}}^{0^{\prime}} T_{2^{\prime}}^{1^{\prime}} T_{3^{\prime}}^{2^{\prime}} T_{p}^{3^{\prime}} T
$$

Here, ${ }_{0^{\prime}}^{0} T$ is:

$$
\begin{gathered}
{ }_{0^{\prime}}^{0} T=\left[\begin{array}{cccc}
\cos \pi & -\sin \pi & 0 & D_{p} \\
\sin \pi & \cos \pi & 0 & 0 \\
0 & 0 & 1 & 0 \\
0 & 0 & 0 & 1
\end{array}\right] \\
0_{1^{\prime}}^{0^{\prime}} T={ }_{1}^{0} T,{ }_{2^{\prime}}^{1^{\prime}} T={ }_{2}^{1} T,{ }_{3^{\prime}}^{2^{\prime}} T={ }_{3}^{2} T,{ }_{p}^{3^{\prime}} T={ }_{p}^{3} T
\end{gathered}
$$

Then, work spaces of both CBHA arms are generated as shown in Fig. 7, at center point of the platform. The intersection of these work spaces is the common work space shown in Fig. 7, this is the work space of center point of platform in which both of arms can reach. But there are infinite number of solutions to get different work space of center point of platform reachable by both of the arms, either by changing length of the platform or by fixing both of the CBHA arms considering different distances between their bases, leading to different amount of volume of common work space. Volume of common work space is calculated using 'alphavol' function of MATLAB as in Fig. 8. Optimization is needed as the volume of work space is different for different configurations.
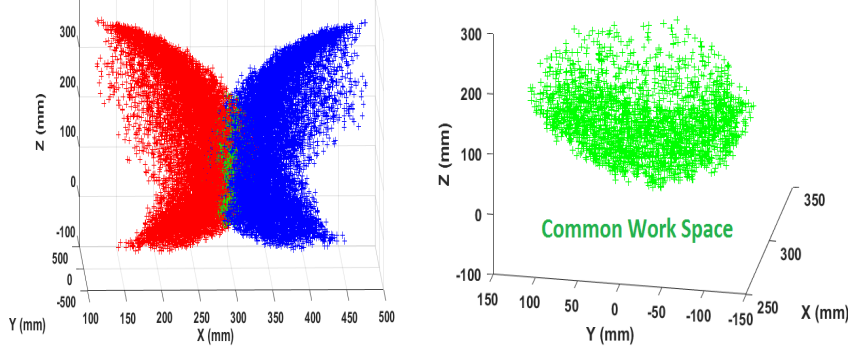

Fig. 7. Work space of both CBHA arms at center point of platform

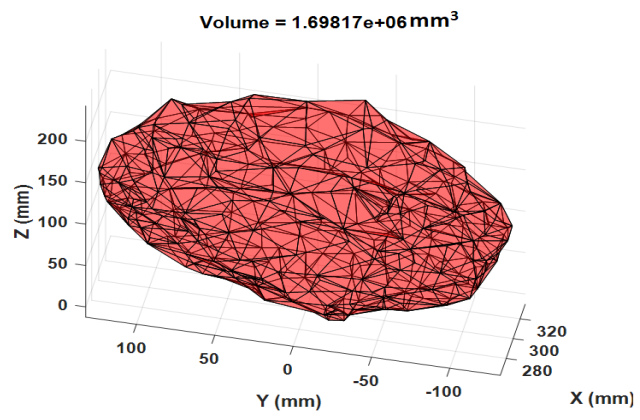

Fig. 8. Volume of common work space

\section{B. Optimal Work Space}

The objective of this paper is to establish a relation between the distance $D_{p}$ between the bases of two arms and the platform length $a$ as shown in Fig. 6, for achieving the maximum volume of common workspace. This is achieved by calculating the platform length for maximum volume of workspace at various $D_{p}$, using optimization technique. Then regression is used to establish a relation between optimal platform length and distance between arms.

For obtaining correct results of optimization, it is necessary to select a proper algorithm for optimization. A general idea of selection of algorithms and the correct variable bounds, can be achieved by observing the general trend of change in optimization function with change in values of optimization variables.

For this problem, the volume of the common workspace is used as the optimization function. On observing the general trend of change in volume of common workspace it is observed that the optimization function is unimodal (i.e. single peak). The trend is checked for various values of $D_{p}$ and the unimodal nature of the optimization function is confirmed as shown in Fig. 9. From Fig. 9 it is also observed that the optimization function shifted in the positive $x$ direction (i.e. higher value of a) on increasing $D_{p}$. To accommodate this change, the variable bounds are selected such that the maxima always lies within the selected bounds. For optimizing a unimodal function depending on a single variable, the region elimination technique is used. This technique can be implemented by using many available methods. In this work, Golden Section Search Method (GSSM) is used. 


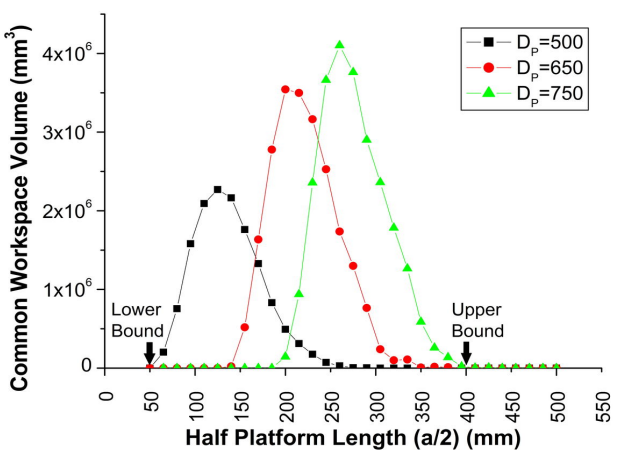

Fig. 9. Variation of volume of common work space w.r.t design parameters of parallel manipulator

Region elimination methods are iterative optimization methods. These methods start by selecting two points within the selected variable bounds. The function is evaluated at the selected points. The results are compared to recognize the point of higher and lower value of optimization function. The variable bound near the point of lower value is shifted to that point; hence, the point of lower function value will act as a new variable bound for the next iteration. The bound can be shifted because in a unimodal function, the maxima can not lie between these points. The iterations are repeated until a predefined termination criterion is met.

The golden section search method is used for selecting the two points inside the variable bounds for every iteration. In this method the search space is mapped linearly to lie between 0 and 1 . The two points are selected such that they are at a distance $t(=0.618)$ from each end. So the eliminated search space has a length of $(1-t)$ i.e. 0.382 . Hence in every iteration $38.2 \%$ of region is eliminated. Another advantage of using golden section search method for selection of points is that in every new iteration one of the two new points is same as that from the previous iteration. Therefore, function needs to be evaluated only once for completing the new iteration.

\section{Results}

The optimized half lengths $(a / 2)$ are plotted against their respective distance between robots $\left(D_{p}\right)$ as in Fig. 10. The trend shows a nearly linear variation. Therefore a linear approximation is performed to obtain a mathematical relationship between distance between robots and optimal platform lengths.

The obtained relation is:

$$
a / 2=0.5286 D_{p}-136.2687
$$

Therefore,

$$
a=1.0572 D_{p}-272.5374
$$

The approximated relation is checked against the obtained results for errors as shown in Fig. 11. Both the absolute and percentage error are well within the acceptable limits. The max absolute error and max percentage error are $14.77 \mathrm{~mm}$ and $5.56 \%$ respectively. This error can further be reduced by

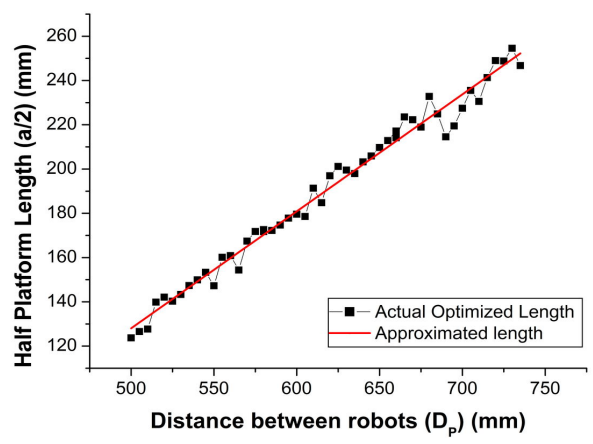

Fig. 10. Curve fitting for approximation of optimized platform length

using more iterations for obtaining the optimal half lengths.

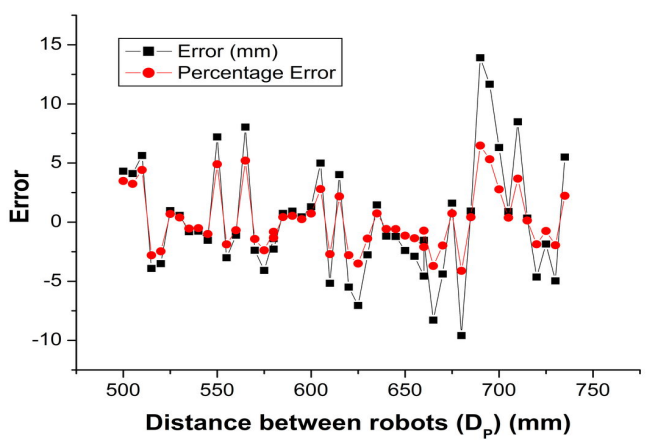

Fig. 11. Error analysis of optimal platform size

\section{Parallel Continuum Manipulator Designed USING THREE CBHA ARMS}

\section{A. Work Space Identification}

A parallel continuum manipulator using three CBHA arms can be created as shown in Fig. 12. The tips of three CBHAs are joined to a common triangular platform through spherical joints. This triangular platform is supposed to be in the form of equilateral triangle. Centroid of triangular platform $(p)$ is at distance $a$ from the end points of CBHAs. Fig. 13 represents the schematic diagram of planar base of this setup, a triangle $\mathrm{ABC}$ is formed joining the bases of three CBHAs. The CBHAs are placed at the edges of the triangle $\mathrm{ABC}$, facing in the direction of arrows, bisecting the angles of triangle. The CBHA2 and CBHA3 are placed at a distance of $D_{P 1}, D_{P 2}$ respectively and at angles $\theta_{1}, \theta_{2}$ respectively from the CBHA1.

Same as previous case, using transformations, the work spaces of all three CBHAs are generated till their tip points and then they are extended to the center point $p$ of the platform. Work space of center point of platform is computed using intersection of work spaces of three CBHAs as shown in Fig. 14. Therefore, this is the work space where all three 


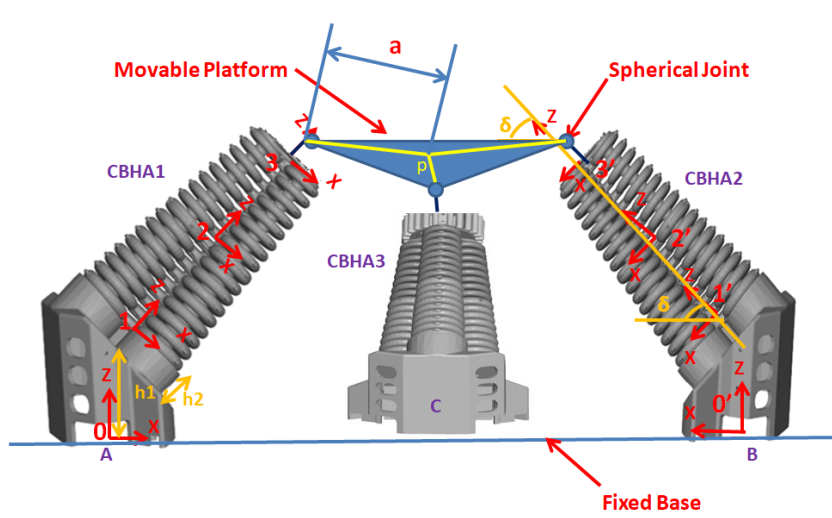

Fig. 12. Set-up for parallel continuum manipulator using three CBHAs

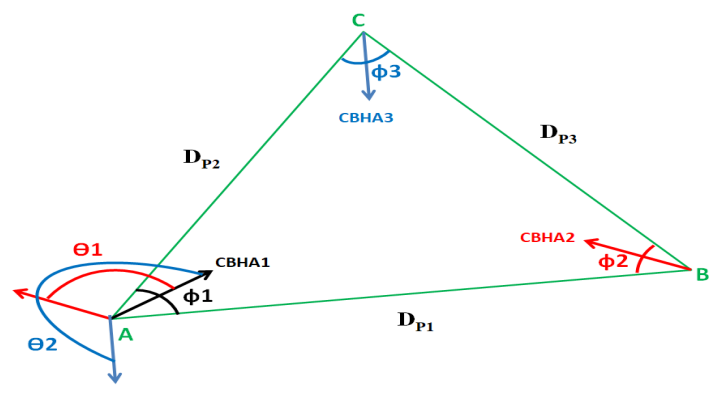

Fig. 13. Schematic diagram of planer base of set-up

CBHAs can reach. Volume of this work space is computed using 'alphavol' function of MATLAB same as previous case. In this case also, there are a lot of combinations of
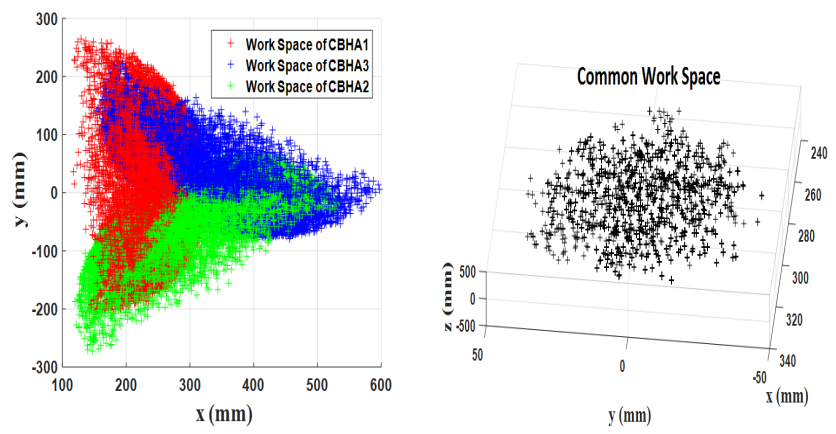

Fig. 14. Work space of three CBHA arms at center point of platform

placement of CBHAs to design a parallel continuum manipulator. Therefore, a relation is needed to develop between $D_{P 1}, D_{P 2}, D_{P 3}$ and distance from tip of CBHA to center of platform $(a)$, using optimization of volume of common work space. First of all, the geometrical relation between parameters are computed using Fig. 13 as:

$$
\begin{aligned}
& \phi_{1}=\cos ^{-1}\left(\frac{D_{P 2}^{2}+D_{P 1}^{2}-D_{P 3}^{2}}{2 D_{P 1} D_{P 2}}\right) \\
& \phi_{2}=\cos ^{-1}\left(\frac{D_{P 1}^{2}+D_{P 3}^{2}-D_{P 2}^{2}}{2 D_{P 1} D_{P 3}}\right)
\end{aligned}
$$

$$
\begin{gathered}
\phi_{3}=\cos ^{-1}\left(\frac{D_{P 2}^{2}+D_{P 3}^{2}-D_{P 1}^{2}}{2 D_{P 2} D_{P 3}}\right) \\
\theta_{1}=\frac{\phi_{3}+180^{\circ}}{2} \\
\theta_{2}=\frac{540^{\circ}-\phi_{2}}{2}
\end{gathered}
$$

So for the values of $D_{P 1}, D_{P 2}, D_{P 3}$, the above relations can be used to compute angles $\theta_{1}, \theta_{2}$ to place other two CBHAs with respect to CBHA1.

\section{B. Optimal Work Space}

In this case a relation between the distances $D_{p 1}, D_{p 2}, D_{p 3}$ between the bases of three arms and the platform length $a$ for achieving the maximum volume of common workspace is needed. As the platform is in the form of equilateral triangle, using optimal value of $a$, triangular platform can be designed. Using the same approach as previous case, the variation of the volume of the common workspace due to different sizes of platform is calculated. The variation is plotted for one condition i.e. $D_{p 1}=D_{p 2}=D_{p 3}=300 \mathrm{~mm}$, for selecting the correct optimization algorithm. The plot is shown in Fig. 15 , from the plot it is observed that the variation is not unimodal. It means in this case simple algorithms like in previous case can not be used to find the optimal value. Here, to find the global optimal condition population based methods are required. For this case genetic algorithm is used to find the global optimal condition. The volume (volume of work space) tolerance for finding the optimal size is set to $10 \mathrm{~mm}^{3}$.

Genetic algorithm is a method to solve both constrained and unconstrained optimization problems and is inspired from biological evolution. This method can be applied when standard optimization methods are not suitable. Genetic algorithm do repetitions to modify a population of individual solutions. From current population, this algorithm selects individuals at random as parents, for each step. These parents are used to produce the children for the next generation. With successive generations, the population evolve toward an optimal solution.

To create next generation from current population, the genetic algorithm uses mainly three rules:

- Reproduction: It selects the parents, which produce children (population) for next generation.

- Crossover: It combines two parents to form children for the next generation.

- Mutation: It does random changes to parents to produce children.

Therefore, Using genetic algorithm, optimal value of platform size $(a)$ is determined for various combinations of $D_{p 1}$, $D_{p 2}, D_{p 3}$.

\section{Results}

Like the previous case, to establish a relationship between the distances between the bases of CBHAs and the optimum platform size, a database is created. The database is consisted of various combinations of distances $D_{p 1}, D_{p 2}, D_{p 3}$, ranging 


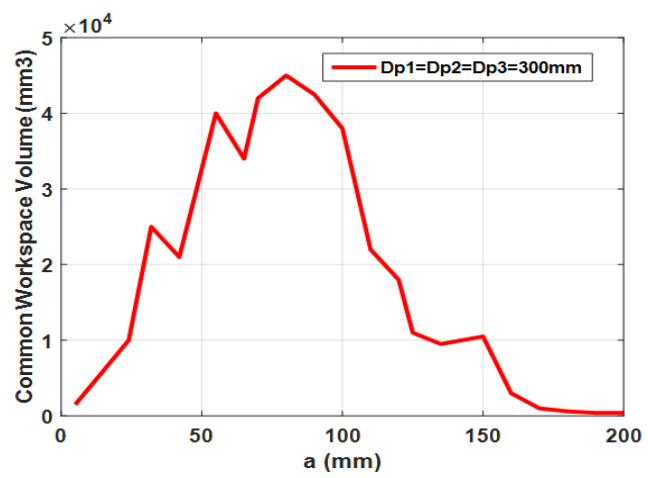

Fig. 15. Variation of volume of Common Work Space w.r.t. design parameters of parallel manipulator with three CBHAs

from $150 \mathrm{~mm}$ to $450 \mathrm{~mm}$. These distances are mapped to their respective optimal platform size, calculated using genetic algorithm. This database is used to establish the relationship using neural network approach.

Artificial neural networks are computing systems inspired by the brain. It is a technique using which the system learns to do tasks by considering known inputs and outputs, generally without any task-specific programming. The network consists of a specific number of hidden layers. The hidden layers consist of many artificial neurons analogous to the axons in the brain. Each hidden layer has weights and biases associated with it. With the correct approximation of these weights and biases, a relationship between the inputs and outputs is established.

In our case, the dataset is divided such that $70 \%$ of data is used for training, $15 \%$ of data is used for validation and $15 \%$ data is used for testing of the network. Neural network consisting 25 hidden layers is trained using the Levenberg-Marquardt algorithm. The trained neural network has coefficient of correlation for training, validation and testing as $0.929,0.936$ and 0.95 respectively. The error analysis in Fig. 16 shows that majority of the absolute error lies within $12 \mathrm{~mm}$ from the 0 -error position. Therefore, the accuracy of the neural network is acceptable.

\section{CONCLUSIONS}

In this paper, an approach for optimal identification of workspace of parallel continuum manipulator, made up of two as well as three CBHAs, is presented. This approach can be extended to other soft or rigid manipulator systems. In case of parallel manipulator consisting of two CBHAs, Golden section search optimization technique helps to establish a relationship between optimal platform length and distance between CBHA arms, to obtain the maximum volume of the work space, as the optimization function was unimodal. But in case of parallel manipulator consisting of three CBHAs, Genetic algorithm (GA) as well as neural network technique are used, as the optimization function was not unimodal. The relationships can be used to get optimal platform length for any distance between base of robotic arms. The same approach can be used for parallel

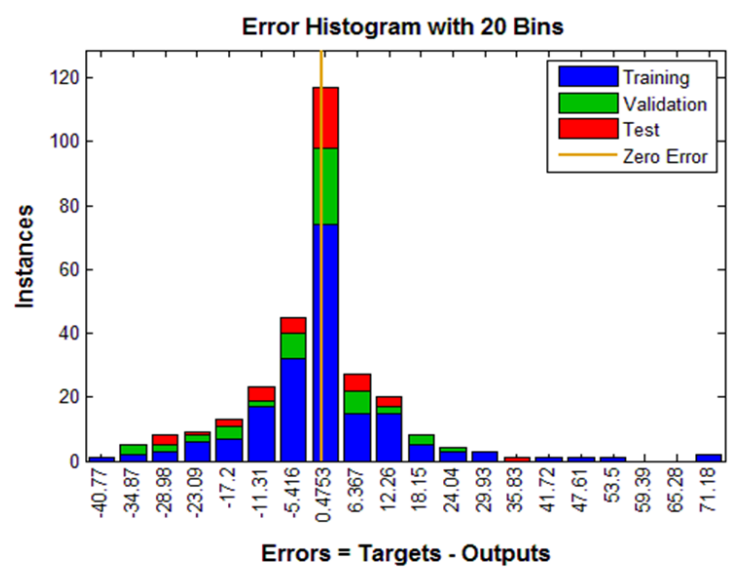

Fig. 16. Error Distribution

continuum manipulator consisting of more number of CBHA manipulators.

\section{ACKNOWLEDGMENT}

This work was supported by the joint Collaborative Research Project between CNRS in France and DST in India (PRC CNRS-DST 2016-2018). The authors want to thank Dr. Reinhard Pittschellis and Dr. Dirk Pensky from Festo Didactic company in Denkendorf (Germany) for their technical assistance and support on RobotinoXT.

\section{REFERENCES}

[1] S. C. Tartari Filho and E. L. L. Cabral, "Kinematics and workspace analysis of a parallel architecture robot: the hexa," in 18th International Congress of Mechanical Engineering, Ouro Preto, MG, 2005.

[2] D. Chablat, R. Jha, F. Rouillier, and G. Moroz, "Workspace and joint space analysis of the 3-rps parallel robot," in Proceedings of ASME, 2014.

[3] X.-J. Wang, C.-H. Zhang, C.-D. Wang, and H. Zheng, "Workspace analysis of a novel parallel robot named 3-r2h2s with three freedoms," 2013.

[4] D. Chablat, L. Baron, and R. Jha, "Kinematics and workspace analysis of a 3ppps parallel robot with u-shaped base," in International Design Engineering Technical Conferences \& Computers and Information in Engineering Conference, 2017.

[5] Z. Gao and D. Zhang, "Workspace representation and optimization of a novel parallel mechanism with three-degrees-of-freedom," Sustainability, vol. 3, no. 11, pp. 2217-2228, 2011.

[6] Y. Hou and Y. Zhao, "Workspace analysis and optimization of 3-puu parallel mechanism in medicine base on genetic algorithm," The open biomedical engineering journal, vol. 9, p. 214, 2015.

[7] C. E. Bryson and D. C. Rucker, "Toward parallel continuum manipulators," in Robotics and Automation (ICRA), 2014 IEEE International Conference on. IEEE, 2014, pp. 778-785.

[8] G. Wu, G. Shi, and Y. Shi, "Modeling and analysis of a parallel continuum robot using artificial neural network," in Mechatronics (ICM), 2017 IEEE International Conference on. IEEE, 2017, pp. 153-158.

[9] C. Escande, T. Chettibi, R. Merzouki, V. Coelen, and P. M. Pathak, "Kinematic calibration of a multisection bionic manipulator," IEEE/ASME transactions on mechatronics, vol. 20, no. 2, pp. 663-674, 2015. 